ISSN 0819-2642

ISBN 0734026307

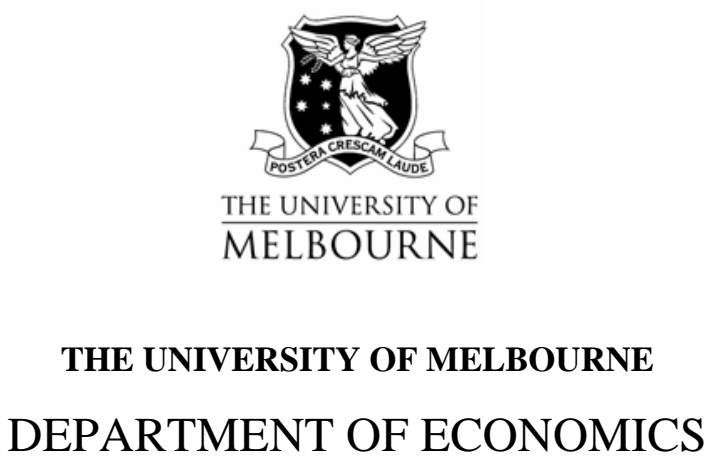

RESEARCH PAPER NUMBER 972

SEPTEMBER 2006

\title{
POPULATION AGEING AND INTERTEMPORAL CONSUMPTION: REPRESENTATIVE AGENT VERSUS SOCIAL PLANNER
}

by

John Creedy

\&

Ross Guest

Department of Economics

The University of Melbourne Melbourne Victoria 3010

Australia. 


\title{
Population Ageing and Intertemporal Consumption: Representative Agent versus Social Planner
}

\author{
John Creedy and Ross Guest \\ University of Melbourne and Griffith University
}

\begin{abstract}
This paper examines the optimal path of consumption over time in the context of population ageing. Older age groups are considered to have relatively greater 'needs', resulting for example from additional health costs. These differences give rise to the concept of the 'equivalent number of persons', as distinct from the population size. Emphasis is given to the difference between a framework involving a representative agent and one in which plans are made by a social planner. The precise conditions under which consumption growth paths are the same under the representative agent and the social planner are established. This equivalence is found to hold only in the case where the social planner's value judgements are such that individuals are considered to be the appropriate unit of analysis. An alternative assumption, in which equivalent persons are regarded as the appropriate units, is found to give rise to a different optimal consumption path. Numerical examples demonstrate the relative orders of magnitude for a range of parameter values. The differences are found to be potentially important. The choice of appropriate consumption units - individuals or equivalent persons - is far from arbitrary since it involves possibly conflicting value judgements. This choice has implications for policies designed to influence the optimal saving rate, such as superannuation policy and the fiscal balance.
\end{abstract}




\section{Population Ageing and Consumption: Representative Agent versus Social Planner}

\section{Introduction}

The aim of this paper is to examine the optimal path of aggregate consumption in the context of population ageing, where older age groups are considered to have relatively greater 'needs', resulting for example from additional health costs. These differences give rise to the concept of the 'equivalent number of persons', as distinct from the population size. Many models in this context employ the concept of a hypothetical representative agent who is assumed to be infinitely lived and who has the characteristics (that is, the 'needs') of an average person in the population in each year. The present paper compares the representative agent approach with an alternative in which the optimal aggregate consumption stream is determined by a social planner who is considered to maximise a social welfare, or evaluation, function defined over the same time horizon. The value judgements of the social planner are made explicit in the form of the welfare function.

Using an individualistic and additive welfare function, total consumption per equivalent person contributes to social welfare in each period. In this preliminary investigation, individuals are considered to differ only by age and hence the planner is not concerned with inequality. One important issue relates to the choice of consumption unit in weighting the consumption per equivalent person in each period. Two cases are examined here - the use of individuals and of equivalent persons. Each of these cases has sensible, but possibly inconsistent, welfare rationales.

The analysis is motivated by the extensive debate regarding the implications of population ageing, and the potential for tax smoothing to achieve an optimal time path of aggregate consumption. The idea that governments should smooth the tax burden over time was first advanced by Barro (1979), who showed that, in a deterministic setting, a flat path of the tax rate over time would minimize the distortions to behaviour arising from taxation. A key insight is that the tax rate must not distort intertemporal consumption choices and must therefore tax consumption at the same 
rate through time in the long run (Chamley, 1986). ${ }^{1}$ Therefore the path of the ratio of optimal income tax to GDP depends on the optimal path of consumption.

Consumption smoothing implies a varying ratio of consumption to income and therefore a varying path of the optimal income tax to GDP ratio. In addition, because the optimal consumption path implies an optimal saving path, the analysis in the present paper has implications for policies designed to affect optimal saving such as superannuation policy and the fiscal balance. It is shown that these policy implications depend on value judgements in the evaluation of social welfare. ${ }^{2}$

The dominant framework for macroeconomic modelling is based on the behaviour of representative agents because the outcomes can be traced to microeconomic foundations. These agents can have either infinite lives, single period lives leading over time to a dynasty of individuals, or finite multi-period lives which imply a number of overlapping generations at a point in time. The origin of this framework was provided by Ramsey (1928) who assumed an infinitely lived individual. It is widely used in modelling economic growth and macroeconomic aggregates. Seminal expositions of a range of these models include, for example, Barro and Sala-i-Martin (1995) and Obstfeld and Rogoff (1996). All large scale multisector, multi-region models (computable general equilibrium models) used to model national and world economies are based on the behaviour of representative agents. Examples include the OECD's MINILINK model, the IMF's MULTIMOD model and the European Commission's QUEST model. ${ }^{3} \mathrm{~A}$ common feature of these models is that the agents optimise intertemporally and this feature is the focus of attention below.

\footnotetext{
${ }^{1}$ In the short run this rule is complicated by the optimal tax rate on the income from capital
} which is 100 percent initially, falling to zero in the long run (Chamley, 1986).

${ }^{2}$ These policy implications have not been acknowledged in Australian Governmentcommissioned analyses of the effects of population ageing. See the Intergeneration Report, Australian Government (2002) and Productivity Commission (2005).

${ }^{3}$ For applications of these models to population ageing see, for example, Werner and Veld (2002) for the QUEST model, Turner et al. (1998) for the MINILINK model, and Faruqee and Muhleisen (2001) for the MULTIMOD model. 
However, the representative agent framework has been subject to a number of strong criticisms; see, for example, Kirman (1992). Also, it has limitations in social welfare analysis of public policies such as fiscal policy where it is useful to know the socially optimal outcome. This is because the representative agent model generates Pareto optimal aggregate outcomes only under strict assumptions; see, for example, Lewbel (1989). For this reason it is sometimes assumed that the economy is run by a benevolent social planner or decision maker.

This paper contributes to the literature by identifying a source of difference between the representative agent and social planner paradigms in the particular context of population ageing. The difference turns out to depend on whether the social planner is concerned with individuals or equivalent persons in evaluating social welfare.

The basic framework of analysis, involving a difference between the number of people and the equivalent population size, is described in section 2 . The optimal consumption path of a representative agent is examined in section 3. Section 4 turns to the optimal path determined by a social planner, where particular attention is given to the choice of the unit of analysis. Some comparisons are made in section 5, followed by numerical examples in section 6 . Brief conclusions are in section 7 .

\section{The Basic Framework}

This section outlines the basic framework of analysis, involving population growth arising from differential growth rates across age groups. The number of individuals aged $i$ in year $t$ is denoted $N_{i, t}$, so the total population in year $t$ is $N_{t}=\sum_{i} N_{i, t}$. Individuals of different ages are assumed to have different 'basic needs', reflected in an equivalence size, $s_{i}$. This is similar in some ways to the type of adult equivalence scale used in the measurement of poverty and inequality. A higher value of $s_{i}$ implies a lower capacity to derive utility from a given dollar amount of consumption. The scale is normalised such that it is unity at age $i^{*}$, so that $s_{i^{*}}=1$. The population size can therefore be adjusted to 'equivalent person' units. The equivalent size at time $t, P_{t}$, is thus: 


$$
P_{t}=\sum_{i} s_{i} N_{i, t}
$$

The average equivalent size per person at time $t$ is given by:

$$
\begin{aligned}
s_{t} & =\sum_{i} s_{i}\left(\frac{N_{i, t}}{N_{t}}\right) \\
& =\frac{P_{t}}{N_{t}}
\end{aligned}
$$

Over time, the population age structure is assumed to change in an exogenous manner, resulting in population growth at the proportional rate, $n_{t}$, where:

$$
n_{t}=\frac{1}{N_{t}} \frac{d N_{t}}{d t}=\sum_{i}\left(\frac{N_{i, t}}{N_{t}}\right)\left(\frac{d N_{i, t}}{d t} \frac{1}{N_{i, t}}\right)
$$

and is a weighted sum of the proportional change in each age group. Similarly, the proportional change in the equivalent population size is given by:

$$
p_{t}=\sum_{i}\left(\frac{P_{i, t}}{P_{t}}\right)\left(\frac{d N_{i, t}}{d t} \frac{1}{N_{i, t}}\right)
$$

Letting $n_{i, t}=\left(\frac{d N_{i, t}}{d t} \frac{1}{N_{i, t}}\right)$, then:

$$
p_{t}-n_{t}=\sum_{i} n_{i, t}\left(\frac{N_{i, t}}{N_{t}}\right)\left(\frac{s_{i}}{s_{t}}-1\right)
$$

and the difference between the growth rates is a weighted average over all age groups of the growth in each age group, with weights depending on the proportional difference between the equivalence scale for that age and the average equivalence size of the population.

Suppose the only changes taking place over time are the exogenous population changes affecting total population and its age composition, and average income changes which result from labour productivity growth at the fixed rate, $g$. Individuals alive at any time have been assumed to differ only in their ages; members of the same cohort have common income and consumption levels. The question considered here concerns the optimal aggregate consumption path associated with the productivity and 
population changes. Clearly, this must depend crucially on the way in which the objective function is specified.

\section{The Representative Agent}

Over time, the average age of the population, and hence its average 'equivalent size', changes. Consider a 'representative agent', who in each period is regarded as having the average age of the population and hence an equivalent size equal to the average equivalent size of the population. This artificial representative person is assumed to maximise a utility function, specified over an infinite horizon, which has as arguments the level of consumption in each period, $c_{t}$, expressed as a ratio of (average) equivalent size in each period. ${ }^{4}$ The representative agent's optimisation problem is to select the time path of consumption, $c_{t}$.

While individuals have finite lifetimes, the representative agent has the age and thus needs of an average person in each period and is assumed to maximise a utility function of the following iso-elastic form:

$$
U=\sum_{t=1}^{\infty}\left[\frac{c_{t}}{s_{t}}\right]^{1-\beta} \frac{(1+\theta)^{1-t}}{1-\beta}
$$

where $\theta$ is the pure time preference rate and $\beta$ is the representative agent's elasticity of marginal utility of consumption. ${ }^{5}$ This is maximised subject to the intertemporal budget constraint, assuming no initial assets, given by:

$$
\sum_{t=1}^{\infty} c_{t}(1+r)^{1-t}=\sum_{t=1}^{\infty} y_{t}(1+r)^{1-t}
$$

${ }^{4}$ The term $c_{t}$ is therefore not an average, but the consumption of the representative agent who has the average needs of the population. This differs from an alternative approach, which may be to define $c_{i, t}$ as the consumption of each person aged $i$ at time $t$, where all individuals of the same age have the same consumption level. Average consumption at $t$ is thus $\frac{1}{N_{t}} \sum_{i} N_{i, t} c_{i, t}$. The representative agent does not, in the approach adopted in the text above, attempt to maximize utility defined in terms of the ratio of average consumption to average equivalent population size.

${ }^{5}$ The parameter $\beta$ may be said to reflect the representative agent's (relative) aversion to consumption variability over time. The interpretation of this is discussed further below. 
where $r$ is the constant interest rate.

The marginal rate of substitution between consumption in periods $t$ and $t+1$, the ratio of marginal utilities, is:

$$
\frac{\partial U / \partial c_{t}}{\partial U / \partial c_{t+1}}=\left(\frac{s_{t}}{s_{t+1}}\right)^{\beta-1}\left(\frac{c_{t+1}}{c_{t}}\right)(1+\theta)
$$

Hence, substituting into the first-order condition for optimal consumption,

$$
\begin{aligned}
& \frac{\partial U / \partial c_{t}}{\partial U / \partial c_{t+1}}=(1+r), \text { gives: } \\
& \frac{c_{t+1}}{c_{t}}=\left(\frac{1+r}{1+\theta}\right)^{\frac{1}{\beta}}\left(\frac{s_{t+1}}{s_{t}}\right)^{\frac{\beta-1}{\beta}}
\end{aligned}
$$

Taking logarithms of both sides and approximating, yields:

$$
\frac{\dot{c}}{c}=\frac{1}{\beta}\left[r-\theta+(\beta-1)\left(\frac{\dot{s}}{s}\right)\right]
$$

Finally, assuming constant labour productivity growth, $g$, the growth rate of consumption per equivalent persons, deflated by productivity, is given by:

$$
\frac{\dot{c}}{c}=\frac{1}{\beta}[r-\theta+(\beta-1)(p-n)]-g
$$

since $s=P / N$ and $\dot{s} / s=p-n$.

\section{The Social Planner.}

This section considers the optimal consumption path determined by a social planner whose aim is to maximise an additive social welfare function defined over an infinite horizon. As it has been assumed that individuals alive at any time differ only in their ages, the social planner has no concern for within-period inequalities. Section 4.1 discusses the basic form of the welfare function, and examines the precise conditions under which welfare can be regarded as a function of the ratio of aggregate consumption to aggregate equivalent population size in each period. The question then remains of the choice of how to weight this term. This involves the choice of appropriate unit of analysis - that is, whether the unit should be the individual or the equivalent person. This issue has been examined in the context of inequality and poverty measurement, where it has been shown that the choice depends on possibly 
conflicting value judgements. These issues are discussed in subsection 4.2.

Subsections 4.3 and 4.4 examine in turn the implications of using the two units.

\subsection{The Social Welfare Function}

Suppose that the planner is concerned with per capita consumption per equivalent person in each period. The term entering the welfare function in each period is thus:

$$
E\left(\frac{c}{s}\right)_{t}=\frac{1}{N_{t}} \sum_{i} N_{i, t}\left(\frac{c_{i, t}}{s_{i, t}}\right)
$$

This may be compared with the ratio of average consumption, $\bar{c}_{t}$, to the average equivalent size of the population, $s_{t}$, which is given by:

$$
\begin{aligned}
\frac{\bar{c}_{t}}{s_{t}}= & \frac{\frac{1}{N_{t}} \sum_{i} N_{i, t} c_{i, t}}{\frac{1}{N_{t}} \sum_{i} N_{i, t} s_{i, t}} \\
= & \frac{C_{t}}{P_{t}}
\end{aligned}
$$

where $C_{t}$ is aggregate consumption at time $t$. Comparison of these two expressions shows that:

$$
E\left(\frac{c}{s}\right)_{t}-\frac{\bar{c}_{t}}{s_{t}}=\frac{1}{N_{t}} \sum_{i} N_{i, t}\left(\frac{c_{i, t}}{s_{i, t}}\right)\left(1-\frac{s_{i, t}}{s_{t}}\right)
$$

In general the ratio of averages and the average of ratios are not equal. Appendix A examines this issue in detail, where it is shown that there are essentially two situations under which the social welfare function may be regarded simply as containing the term $C_{t} / P_{t}$ for each period. The first is the case where, if individuals are considered to attempt to perfectly smooth needs-weighted consumption, $c_{i, t} / s_{i, t}$, such that this term is constant except for some purely random variation over the (finite) lifetime, it may be supposed that $c_{i, t} / s_{i, t}$ is the same for all ages, $i$ (except for a random error term). While the difference in the needs of individuals over the life cycle means that the $c_{i, t}$ vary with $i$, the consumption per equivalent person may be assumed to be constant. In this case the difference above is zero, and the social 
welfare function can be expressed in terms of $C_{t} / P_{t}$ for each period. ${ }^{6}$ The second context is where $s_{i, t}$ and $c_{i, t}$ are uncorrelated, in which case Appendix A shows that the two terms - the ratio of average consumption to average equivalent size and the average of the consumption per equivalent person - are proportional, with the constant of proportionality remaining constant over time. Again, the social welfare function can be expressed in terms of $C_{t} / P_{t}$ for each period.

\subsection{Alternative Consumption Units}

As discussed in the previous subsection, the social welfare function is regarded as a function of consumption per equivalent person in each period. One approach is to treat the individual as the basic unit of analysis, so that the consumption per equivalent person is regarded as being assigned to each of the $N_{t}$ individuals in the population. The value judgement inherent in this approach is that every person 'counts for one' irrespective of the demographic structure of the time period. This approach consequently has the property of anonymity. ${ }^{7}$

However, the use of the individual as the unit of analysis can give rise to a result that may at first seem paradoxical. The equivalence scale implies that a population consisting of a larger proportion of younger individuals is regarded as being 'more efficient' at generating welfare. Hence, a transfer of consumption from a period of low consumption (with many older people having high basic needs) to a richer period (with relatively few older people) may actually raise social welfare. ${ }^{8}$

\footnotetext{
${ }^{6}$ This is proved as follows:

$\frac{C}{P}=\frac{N_{t} \bar{c}_{t}}{\sum_{i} N_{i}\left(\frac{s_{i}}{c_{i}}\right) c_{i}}=\frac{N_{t} \bar{c}_{t}}{\left(\frac{s}{c}\right) \sum_{i} N_{i} c_{i}}=\frac{N_{t} \bar{c}_{t}}{\left(\frac{s}{c}\right) N_{t} \bar{c}}=\frac{c}{S}$

${ }^{7}$ In the context of inequality and welfare measurement, this value judgement was called the 'compensation principle' by Shorrocks (2004) and the 'Pareto indifference principle' by Decoster and
} Ooghe (2003).

${ }^{8}$ Hence the use of individuals does not necessarily satisfy the 'principle of transfers', as shown by Glewwe (1991, p.213) and Decoster and Ooghe (2003). 
An alternative approach to defining a unit of analysis is to use the 'adult equivalent person'. ${ }^{9}$ This approach assigns to each of the $P_{t}$ equivalent persons the consumption per equivalent person. It means that the measurement of consumption and the unit of analysis are treated consistently. Individuals no longer 'count as one' but have a weight depending on the demographic structure of the population to which they belong. An important feature of this approach is that it cannot give rise to the paradoxical situation described above.

Consequently, the choice between individuals and adult equivalents as the basic unit of analysis involves a choice between two incompatible value judgements. They can in principle lead to different conclusions about the effects of transferring consumption between time periods, which has implications for the path of optimal saving and the optimal income tax burden.

\subsection{Individuals as Units of Analysis}

Suppose that the social planner takes the individual as the basic unit of analysis. This implies that $N_{t}$ is used as the weight attached to each period's contribution of consumption per equivalent person to social welfare function. If it is further assumed that the welfare function involves a similar iso-elastic form to that of the representative agent, the welfare function takes the form:

$$
V=\sum_{t=1}^{\infty} N_{t}\left[\frac{C_{t}}{P_{t}}\right]^{1-\beta} \frac{(1+\theta)^{1-t}}{1-\beta}
$$

Here the parameters $\beta$ and $\theta$ refer to the value judgements of the social planner, rather than the utility function of a hypothetical representative individual. ${ }^{10}$ This social welfare, or evaluation, function is maximised subject to the following resource constraint for the economy, again assuming no initial assets:

\footnotetext{
${ }^{9}$ In the context of distributional analyses, this was proposed by Ebert (1997).

${ }^{10}$ The values of $\beta$ and $\theta$ are influenced purely by the value judgements of the social
} planner and therefore cannot be objectively measured. However, attempts to evaluate people's value judgements, or to estimate the values implicit in policy decisions, can be of interest: on these issues, see Creedy (2006). Some writers have clearly expressed their values regarding the choice of $\theta$, believing that it 'should' be zero, but there is certainly no consensus regarding this. 


$$
\sum_{t=1}^{\infty} C_{t}(1+r)^{1-t}=\sum_{t=1}^{\infty} Y_{t}(1+r)^{1-t}
$$

where $Y_{t}$ is aggregate income in period $t$.

The marginal rate of substitution between aggregate consumption in two periods is therefore given by:

$$
\frac{\partial V / \partial C_{t}}{\partial V / \partial C_{t+1}}=\left(\frac{N_{t}}{N_{t+1}}\right)\left(\frac{P_{t}}{P_{t+1}}\right)^{\beta-1}\left(\frac{C_{t+1}}{C_{t}}\right)^{\beta}(1+\theta)
$$

The first-order condition for maximising social welfare is given by $\frac{\partial V / \partial C_{t}}{\partial V / \partial C_{t+1}}=(1+r)$, so that:

$$
\frac{C_{t+1}}{C_{t}}=\left(\frac{1+r}{1+\theta}\right)^{\frac{1}{\beta}}\left(\frac{N_{t+1}}{N_{t}}\right)^{\frac{1}{\beta}}\left(\frac{P_{t+1}}{P_{t}}\right)^{\frac{\beta-1}{\beta}}
$$

Using $C_{t}=\bar{c}_{t} N_{t}$ this condition can be written in terms of changes in average consumption as follows:

$$
\frac{\bar{c}_{t+1}}{\bar{c}_{t}}=\left(\frac{1+r}{1+\theta}\right)^{\frac{1}{\beta}}\left(\frac{N_{t+1}}{N_{t}}\right)^{\frac{1}{\beta}-1}\left(\frac{P_{t+1}}{P_{t}}\right)^{-\left(\frac{1}{\beta}-1\right)}
$$

Taking logarithms and allowing for productivity growth gives:

$$
\frac{\dot{\bar{c}}}{\bar{c}}=\frac{1}{\beta}[r-\theta+(\beta-1)(p-n)]-g
$$

This equation is the same as the result regarding the representative agent, except that the former is expressed in terms of the consumption in each period of a hypothetical infinitely-lived individual who in each period has needs determined by the average equivalent size of individuals. The optimal consumption path is the same for both cases - where the social planner's value judgements regarding $\beta$ and $\theta$ are the same as the assumed preferences of the representative agent.

In the above approach, the social welfare function has been expressed in terms of consumption (per equivalent person) in each period, where the social planner is assumed to be averse to variability over time. This aversion is reflected in the parameter $\beta$, which applies in addition to the pure time preference of the planner, measured by $\theta$. An alternative approach might express the social welfare function in terms of total utility in each period, given an additional assumption that all individuals have the same preferences. For example, suppose individuals have a common iso- 
elastic utility function, with parameter $\beta_{0}$, and the planner's aversion to variability is given by $\beta_{1}$. The above social welfare function then becomes:

$$
V=\sum_{t=1}^{\infty} N_{t}\left(\frac{1}{1-\beta_{0}}\left[\frac{C_{t}}{P_{t}}\right]^{1-\beta_{o}}\right)^{1-\beta_{1}} \frac{(1+\theta)^{1-t}}{1-\beta_{1}}
$$

However, it is found that the Euler equation governing the growth of average consumption is the same as that given above in equation (20), where $\beta$ is replaced using:

$$
\beta=\beta_{1}+\beta_{0}\left(1-\beta_{1}\right)
$$

Hence comparisons can be made simply by reinterpreting the parameter $\beta$.

\subsection{Equivalent Persons as Units.}

Assume that the social planner regards equivalent persons, rather than

individuals, as the appropriate unit of analysis. The weight in each period is thus $\mathrm{P}_{t}$, rather than $\mathrm{N}_{\mathrm{t}}$. The social welfare function becomes:

$$
V=\sum_{t=1}^{\infty} P_{t}\left[\frac{C_{t}}{P_{t}}\right]^{1-\beta} \frac{(1+\theta)^{1-t}}{1-\beta}
$$

The first order condition for aggregate consumption is given by:

$$
\frac{C_{t+1}}{C_{t}}=\left(\frac{1+r}{1+\theta}\right)^{\frac{1}{\beta}}\left(\frac{P_{t+1}}{P_{t}}\right)
$$

Converting to average consumption and allowing for productivity growth gives:

$$
\frac{\dot{\bar{c}}}{\bar{c}}=\frac{1}{\beta}(r-\theta)+(p-n)-g
$$

This Euler equation differs from that in the previous case where the individual is regarded as the appropriate unit of analysis. The difference between the two growth rates of consumption, $\left.\frac{\dot{\bar{c}}}{\bar{c}}\right|_{N}-\left.\frac{\dot{\bar{c}}}{\bar{c}}\right|_{P}$, is given by:

$$
\left.\frac{\dot{\bar{c}}}{\bar{c}}\right|_{N}-\left.\frac{\dot{\bar{c}}}{\bar{c}}\right|_{P}=\frac{1}{\beta}(n-p)
$$

Hence the relative growth rates of consumption depend on the growth rate of the population compared with its equivalent size. For example, if population ageing makes $n<p$ the growth rate of average consumption is lower if the individual is 
regarded as the unit, compared with the case where the equivalent person is the unit. This means that relatively more consumption is moved into earlier periods, when the population is younger and more efficient at converting consumption into welfare. Each individual 'counts as one', so more weight is given to these periods than when the unit is the number of equivalent persons.

\section{Some Comparisons}

The previous sections have derived alternative Euler equations governing the optimal consumption path of the economy. The form of the Euler equation for an infinitely lived representative agent, having in each period the average equivalent size of the population, was found to be the same as that for a social planner who regards the individual as the unit of analysis, or weight, in the social welfare function. ${ }^{11}$ However, if the equivalent person is regarded as the appropriate unit of analysis, the social planner's optimal consumption path differs from the other two cases. As discussed above, the choice of these consumption units, or population aggregates, is a normative issue, involving possibly conflicting value judgements.

The Euler equations differ in the way that the term $(p-n)$ affects the optimal consumption path. The term $(p-n)$ reflects the changing consumption demands, and therefore capacity for generating utility, implied by an ageing population. As the population ages, $P$ rises relative to $N$ and therefore $(p-n)>0$, reflecting increasing consumption demands and declining capacity to generate utility.

To the representative agent and the social planner using the individual as the unit of analysis, $(p-n)>0$ implies an additional subjective discounting factor. If a dollar of consumption is postponed one period it will reduce welfare because the demands on a dollar of consumption are higher. This creates a desire to shift consumption towards the present. On the other hand there is an offsetting desire to shift consumption towards the future which arises from the desire to smooth $C / P$. The desire to smooth consumption is stronger the larger is the value of $\beta$. These two

\footnotetext{
${ }^{11}$ The only difference is that the representative agent Euler equation is in terms of the consumption of the agent in each period, and that for the social planner is in terms of per capita consumption.
} 
opposite forces on intertemporal consumption are reflected in the term $(\beta-1)(p-n)$ in the Euler equation. Hence the net rate of subjective discounting is $\theta-(\beta-1)(p-n)$, which is compared with the interest rate, $r$, to determine whether consumption is tilted toward the future or the present.

To see this effect on consumption tilting, differentiate (20) with respect to the term $(p-n)$.

$$
\frac{\partial}{\partial(p-n)}\left(\frac{\dot{\bar{c}}}{\bar{c}}\right)=\frac{\beta-1}{\beta}=1-\phi
$$

where $\phi=1 / \beta$ is the intertemporal elasticity of substitution. This equation implies that the increasing average consumption demands implied by an ageing population tilts consumption towards the present if $\phi>1$, towards the future if $\phi<1$, and neither way if $\phi=1$.

For the social planner adopting equivalent persons as units, the term $(p-n)$ implies no additional discounting motive because in deriving social welfare the planner is summing over $P$ rather than over $N$. In other words, the growth of $P$ relative to $N$ has no relevance for the evaluation of social welfare. It has relevance only in determining the optimal growth of per capita consumption.

\section{$6 \quad$ Numerical Examples}

This section considers whether the difference between the two approaches using individuals or equivalent persons as units - is important. Numerical examples are given, and sensitivity analyses are reported for alternative values of $\beta$ and projections of $p-n$ using Australian data for the period 2004-2050. Subsection 6.1 examines population growth and the associated changes in the equivalent population size, using a flexible specification for the variation in $s_{i}$ with age. The sensitivity of the optimal consumption stream to variations in the parameters describing the value judgements of the social planner is examined in subsection 6.2. 


\subsection{Population Projections}

The population projections are taken from the Australian Bureau of Statistics (Catalogue 3222.0) series for total persons (as no distinction is made in the model between males and females). These data provide the series for $N$ and therefore $n$.

In calibrating the age-specific consumption weights, $s_{i}$, which are used to calculate the series for $P$ and hence $p$, it is desirable to specify these in terms of a parametric function relating the scales to age. This allows for sensitivity analyses to be carried out. The following semi-logarithmic functional form:

$$
s_{i}=a+\frac{1}{b} \log (i)
$$

is sufficiently flexible and allows the scale to increase systematically with age.

Initial, or 'baseline', parameter values were estimated using values of $s_{i}$ which were calculated as a weighted average of private and social consumption weights. ${ }^{12}$ The private weights were calculated from Household Expenditure Survey data for 2003-4 (Australian Bureau of Statistics, Catalogue 6530.0, Table 19). Expenditure per equivalent adult was calculated by dividing household expenditure for each age category by the number of equivalent adults in the household. The latter were calculated using the parametric equivalence scales suggested by Cutler and Katz (1992) and used by, for example, Banks and Johnson (1994), Jenkins and Cowell (1994). This takes the form:

$$
m=\left(N_{a}+\theta N_{c}\right)^{\alpha}
$$

where $m$ is the number of equivalent adults in the household, $N_{a}$ and $N_{c}$ are the number of adults and children respectively. The parameter $\theta$ is a weight between zero and 1 reflecting the lower consumption needs of children, and $\alpha$ is a parameter reflecting economies of scale in household budgets. The following examples use values of $\theta$ and $\alpha$ of 0.5 and 0.75 respectively; these are in the middle of the range

\footnotetext{
${ }^{12}$ In the context of the social planner, these weights may perhaps be regarded as being
} determined by value judgements concerning, for example, the allocation of health resources to the aged. The sensitivity analyses reported below may thus be regarded as reflecting differing value judgements. 
reported by Creedy and Sleeman (2005), estimated using a wide range of scales suggested in the literature.

The social expenditure weights were calculated using data on age-specific health, education and aged care consumption for 2004 used in Productivity Commission $(2005)^{13}$. Both the private and social consumption weights are assumed here to be constant over the projection period. The total consumption weights, $s_{i}$, were calculated by adding the private and social consumption weights, which are themselves weighted by the shares of private and social consumption in total consumption. The baseline values of $a$ and $b$ respectively were found to be 0.5 and 4.0. Figure 1 shows the profiles obtained using several parameter combinations.

Figure 1 Consumption Weights, $s_{i}$, by Age

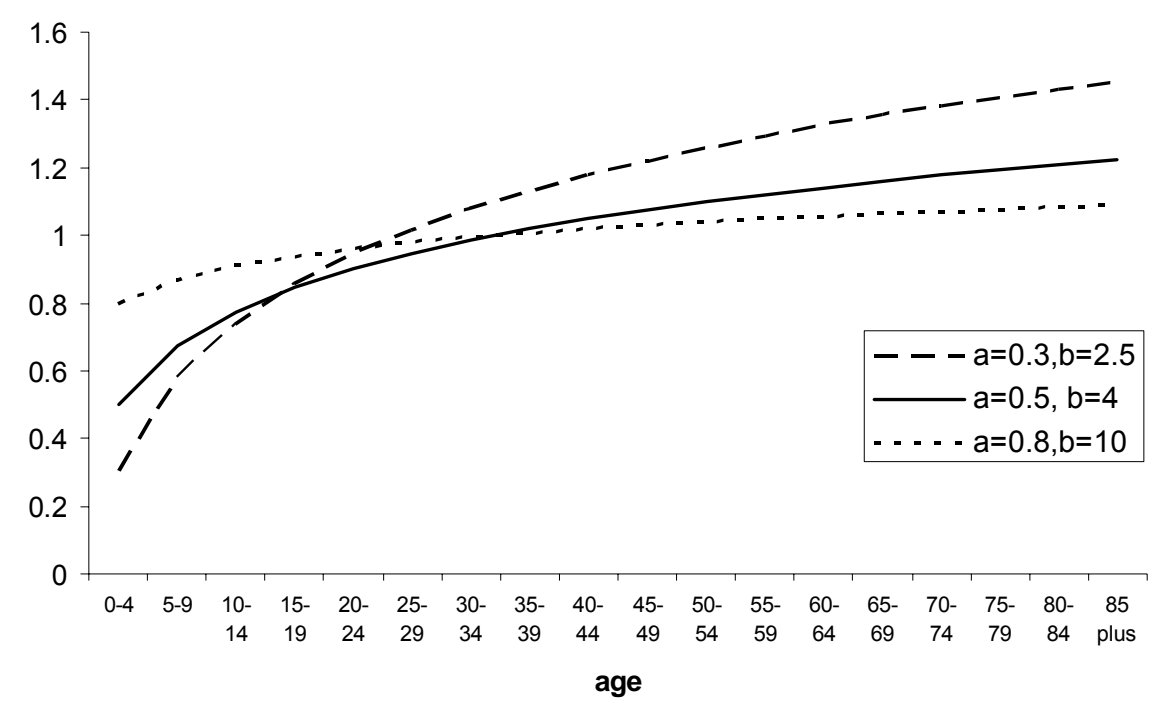

${ }^{13}$ These data were kindly provided by officers at the Productivity Commission. 
Figure 2 Series For $p-n$

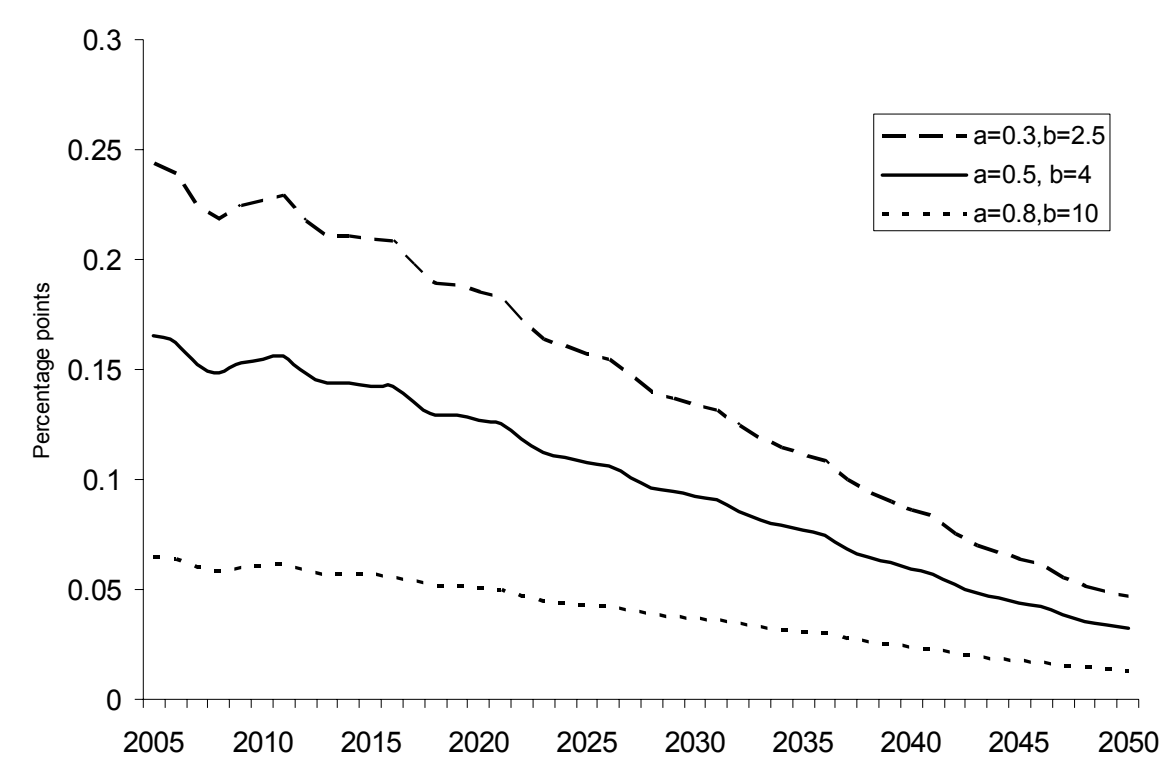

The corresponding series for $p$ - $n$ are plotted in Figure 2. The difference in these growth rates tends to decline over time because the changes in the population age shares diminish over time. For example, in the initial years from 2005 there is a relatively large shift in the population from young to old age groups which tends to produce a large gap between $p$ and $n$. As the age distribution stabilises the gap between $p$ and $n$ diminishes.

\subsection{Optimal Consumption Paths}

Simulating the path of optimal consumption growth, $\dot{c} / c$, requires, in addition to the population series for $n$ and $p$, values of the parameters $r, \beta, \theta$ and $g$. In all the following computations, the values of $r$ and $g$ are fixed at 4 per cent and 1.75 per cent. The Productivity Commission (2005) adopts a base case value of 1.75 per cent for $g$, and 4 per cent is a typical value for the real interest rate long run macroeconomic models.

Three values of $\beta$ are examined: $0.5,1.5$ and 2.5 , which covers a wide range of plausible values for this parameter. It is useful to consider the precise nature of the comparisons being made. When Atkinson (1970), introduced his inequality measure 
based on the welfare function, $W=\sum_{i} \frac{y_{i}^{1-\varepsilon}}{1-\varepsilon}$, he recognised the difficulty of forming views about the orders of magnitude of $\varepsilon$. In order to help interpretation, he used the idea of a 'leaky bucket' experiment, which considers the extent to which a judge is prepared to tolerate some loss in making a transfer from one person to another. Consider two individuals, so that from the welfare function, setting the total differential equal to zero gives:

$$
-\left.\frac{d y_{1}}{d y_{2}}\right|_{W}=\left(\frac{y_{1}}{y_{2}}\right)^{\varepsilon}
$$

The welfare function is thus homothetic, as the slopes of social indifference curves are the same along any ray drawn through the origin. Consider two individuals and, using discrete changes, suppose a dollar is taken from the richest, such that $\Delta y_{2}=-1$. The amount to be given to the other individual to keep social welfare unchanged is thus:

$$
\Delta y_{1}=\left(\frac{y_{1}}{y_{2}}\right)^{\varepsilon}
$$

For example, if $y_{2}=2 y_{1}$ and $\varepsilon=1.5$, it is necessary to give person 1 only 35 cents - a leak of 65 cents from the original dollar taken from person 2 is tolerated. If $\varepsilon=1$, a leak of 50 cents is tolerated. In the present intertemporal context (with an unchanged population), suppose that total consumption in the first period is 100 and this grows at a rate of 0.02 per period. In period 10 it is thus 119.5 , and a judge with $\beta=2$ would be prepared to take a dollar from period 10 , and give only $\$ 0.70$ to period 1. By period 20 total income would be 145.7, and the same judge would reduce period 20's income by $\$ 1$ while adding only $\$ 0.47$ to the first period.

The discount rate, $\rho$, is given by the Ramsey equation, $\rho=\theta+\beta g$. In the following calculations, values of $\theta$ are given by the condition that $\dot{c} / c=0$ in a steady state. This condition is required in non-overlapping generations models, given a constant interest rate, in order to rule out inadmissible paths - in particular paths in which consumption goes to zero or infinity. ${ }^{14}$ This implies that the discount rate is

${ }^{14}$ In overlapping generations models this condition on $\theta$ is not required, because the crosssection profile of consumption in the economy is stable; see Obstfeld and Rogoff (1996, p. 136). 
equal to $r$, so that $\theta=r-\beta g$. Hence $\theta$ is not set independently and is determined by the value of $\beta$ adopted. The resulting series for $\dot{c} / c$ are given in Figures 3 and 4 . Figure 3 applies to the case of the representative agent or the social planner using individuals as the unit of analysis. Figure 4 applies to the case of the social planner using equivalent persons as the unit of analysis.

\section{Figure 3 Optimal Consumption Growth for Social Planner: Individuals as Unit of Analysis}

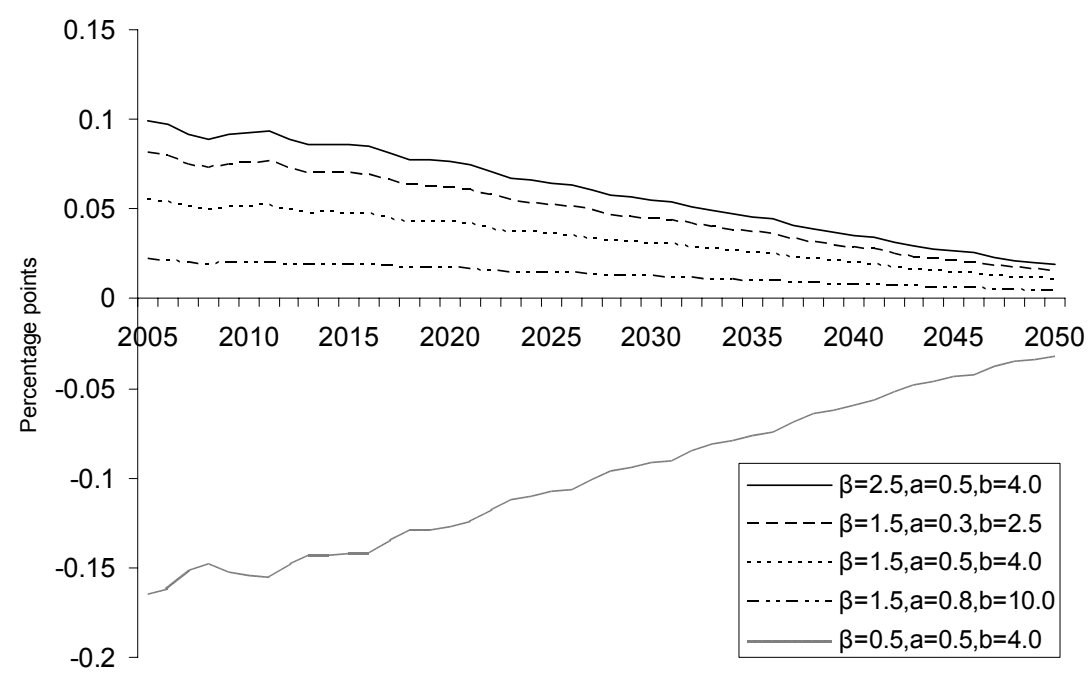

The magnitudes of the differences between the paths in Figures 3 and 4 are indicated in Figures 5 and 6. Figure 5 shows $\left.\frac{\dot{\bar{c}}}{\bar{c}}\right|_{N}-\left.\frac{\dot{\bar{c}}}{\bar{c}}\right|_{P}=-\left(\frac{1}{\beta}\right)(p-n)$, which is the difference in optimal consumption growth, for the various alternative combinations of parameters. Different growth rates imply different paths of consumption in levels. The paths of consumption in levels can be compared by solving for the initial value of consumption for the case where equivalent persons are used, $\dot{\bar{c}} /\left.c\right|_{P}$, that gives the 
same value of aggregate discounted consumption as in the case where the number of person is used, $\dot{\bar{c}} /\left.c\right|_{N}$. This is shown in Figure 6 , where $c^{N}$ and $c^{P}$ refer to levels of consumption where individuals and equivalent persons, respectively, are the unit of analysis. The largest difference occurs for $\beta=0.5$. In that case optimal consumption would be 4 percent higher in 2005 and 4 percent lower in 2050 if individuals are the unit of analysis rather than equivalent persons, for a given value of aggregate discounted consumption over the 46 year period. This is not trivial, nor arguably are the values around 1 and 2 percent for the other simulations.

\section{Figure 4 Optimal Consumption Growth For Social Planner: \\ Equivalent Persons as Unit of Analysis}

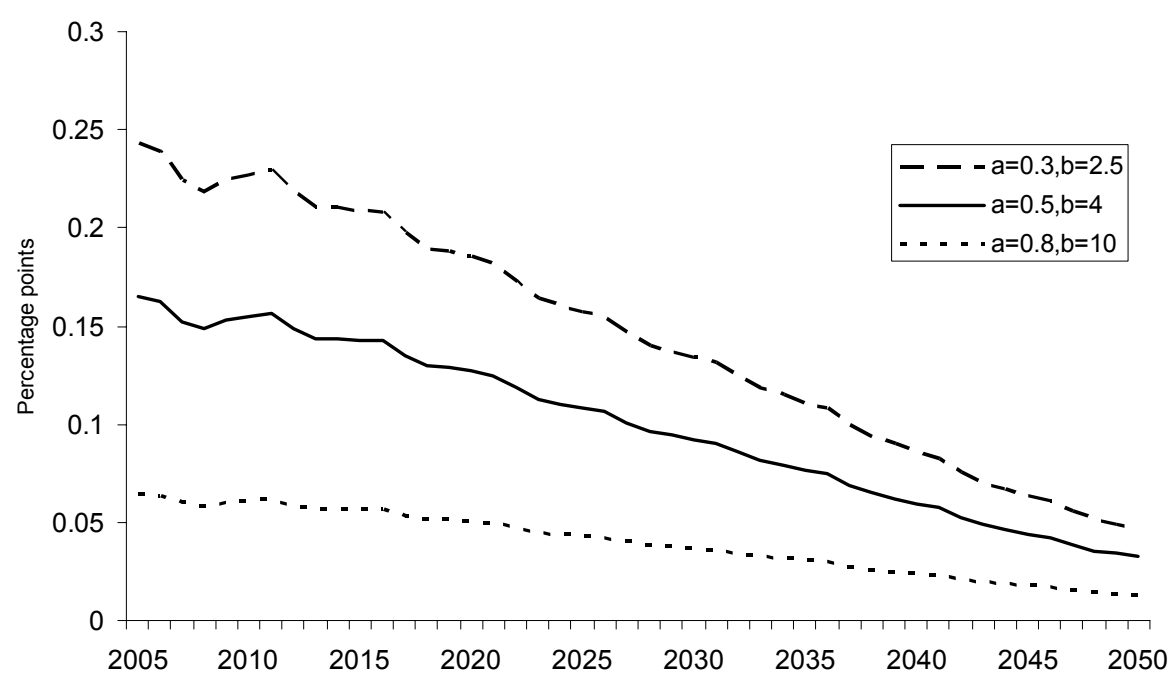




\section{Figure 5 Difference Between Optimal Consumption Paths}

$$
\left.\frac{\dot{\bar{c}}}{\bar{c}}\right|_{N}-\left.\frac{\dot{\bar{c}}}{\bar{c}}\right|_{P}=-\left(\frac{1}{\beta}\right)(p-n)
$$

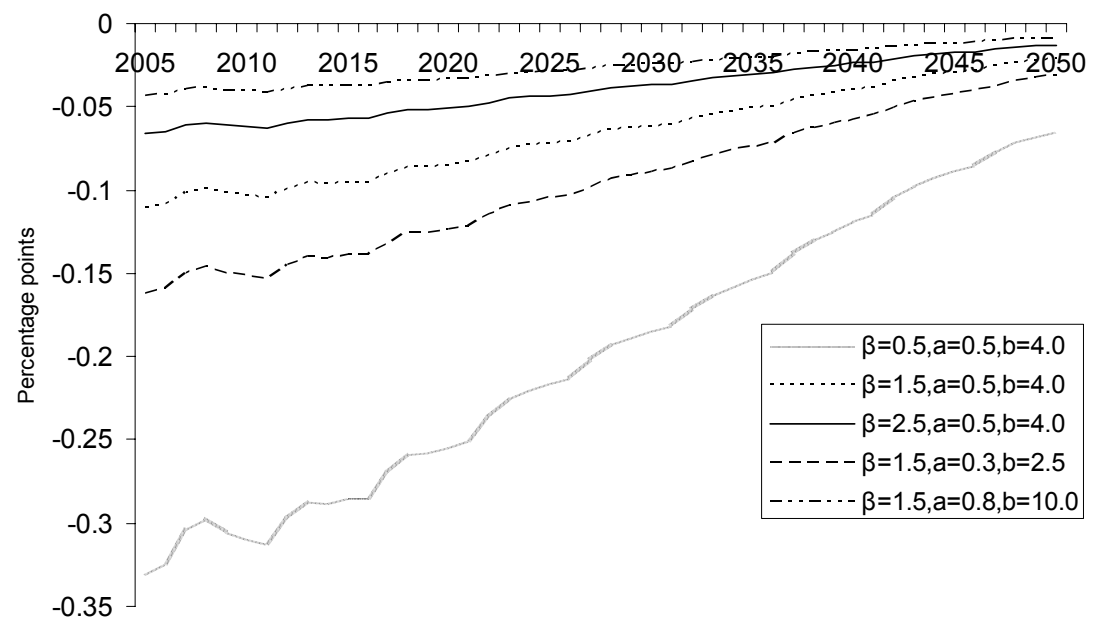

Optimal consumption levels imply optimal saving levels which inform policy decisions such as the appropriate fiscal balance and superannuation policy. If optimal consumption is higher for one social welfare function than another, then optimal saving is lower. In general, if $c^{N}=(1+x) c^{P}$ and assuming income, $\mathrm{y}$, is the same for both consumption paths ${ }^{15}$, then $\frac{s^{N}}{y}=\frac{s^{P}}{y}-x\left(1-\frac{s^{P}}{y}\right)$. For example, if $\frac{s^{P}}{y}=0.2$ and $x=0.01$, a mid-range value in Figure 6 , then $\frac{s^{N}}{y}==0.192$. That is a difference of 0.8 percent in the optimal saving to income ratio which, again, is arguably not trivial.

\footnotetext{
${ }^{15}$ It differs slightly because different consumption paths imply different saving paths and
} hence differen amounts of capital income. 


\section{Figure 6 Percentage Difference in Consumption Levels}

\section{for Given Present Value of Consumption Over the Period}

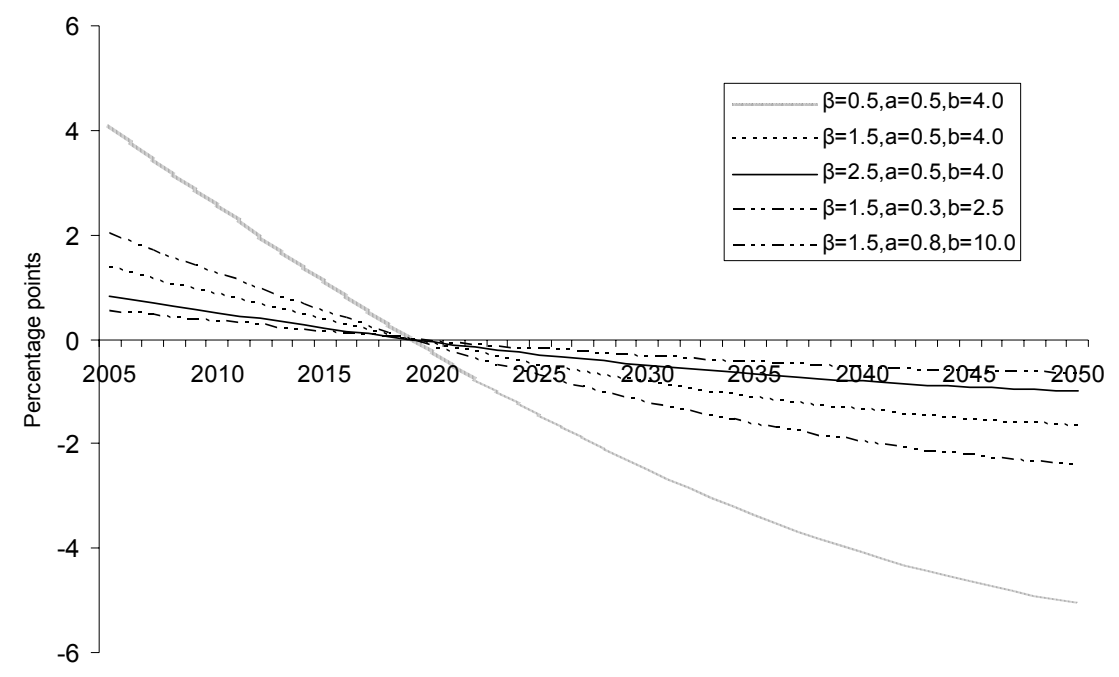

\section{$7 \quad$ Conclusions}

This paper has examined the optimal path of consumption over time in the context of population ageing. Emphasis was given to the difference between a framework involving a representative agent and one in which plans are made by a social planner. The precise conditions under which consumption growth paths are the same under the representative agent and the social planner were established. This equivalence was found to hold only in the case where the social planner's value judgements are such that individuals are considered to be the appropriate unit of analysis. An alternative assumption, in which equivalent persons are regarded as the appropriate units, was found to give rise to a different optimal consumption path. Numerical examples demonstrated the relative orders of magnitude for a range of parameter values. The differences were found to be potentially important. The choice of appropriate consumption units - individuals or equivalent persons - is far from arbitrary since it involves possibly conflicting value judgements. 
These value judgements, by influencing the optimal path of consumption in response to population ageing, have implications for policies designed to influence the optimal saving rate, such as superannuation policy and the fiscal balance. The numerical illustrations reported in this paper suggest that the choice of social welfare function could imply a difference in optimal saving at a given time in the order of 1 percent of GDP, which is arguably not trivial. In addition, the optimal consumption path has implications for the optimal path of income taxes, on the principal that optimal income taxes should not distort intertemporal consumption choices. 


\section{Appendix A. Consumption per Adult Equivalent Person}

This appendix examines the social welfare function expressed in terms of average consumption per equivalent person, and the relationship between a welfare function in terms of the ratio of average consumption to the average equivalent size. In terms of the average ratio of consumption to equivalent size, welfare is:

$$
V=\sum_{t=1}^{\infty} N_{t}\left[E\left(\frac{c_{i, t}}{s_{i, t}}\right)\right]^{1-\beta} \frac{(1+\theta)^{1-t}}{1-\beta}
$$

Writing $c_{i, t}=c_{i}\left(\bar{c}_{t}\right)$ and differentiating the above with respect to average consumption gives:

$$
\frac{\partial V}{\partial \bar{c}_{t}}=N_{t}\left(\frac{1}{\bar{c}_{t}}\right) E\left(\frac{c}{s}\right)_{t}^{-\beta}(1+\theta)^{1-t} E_{W}\left(\frac{c}{s}\right)_{t}
$$

The last term in this expression is a weighted average of $\mathrm{c} / \mathrm{s}$, with weights depending on the elasticity of consumption in each age group with respect to average consumption. Thus:

$$
E_{W}\left(\frac{c}{s}\right)=\frac{1}{N_{t}} \sum_{i} N_{i, t}\left(\frac{\partial c_{i, t}}{c_{i, t}} \frac{\overline{c_{t}}}{\partial \bar{c}_{t}}\right)\left(\frac{c_{i, t}}{s_{i, t}}\right)
$$

This approach is too cumbersome, and it is therefore necessary to consider the relationship between $E(c / s)$ and $E(c) / E(s)$ in more detail. For this purpose it is more convenient to work in terms of distributions.

Suppose $c_{i, t}$ and $s_{i, t}$ are jointly lognormally distributed with means and variances of logarithms denoted by $\mu$ and $\sigma^{2}$, with appropriate subscripts, and with a correlation of $\rho$. Then, dropping the $t$ subscripts, and using the lognormal property that in general $E(x)=\exp \left(\mu_{x}+0.5 \sigma_{x}^{2}\right)$ :

$$
\frac{E(c)}{E(s)}=\exp \left\{\mu_{c}-\mu_{s}+0.5\left(\sigma_{c}^{2}-\sigma_{s}^{2}\right)\right\}
$$

However, $c / s$ is distributed lognormally as: 


$$
\Lambda\left(\mu_{c}-\mu_{s}, \sigma_{c}^{2}+\sigma_{s}^{2}-2 \rho \sigma_{c} \sigma_{s}\right)
$$

Hence:

$$
E\left(\frac{c}{s}\right)=\exp \left\{\mu_{c}-\mu_{s}+0.5\left(\sigma_{c}^{2}+\sigma_{s}^{2}-2 \rho \sigma_{c} \sigma_{s}\right)\right\}
$$

Dividing and rearranging gives the result that:

$$
\frac{E(c) / E(s)}{E(c / s)}=\exp \left\{\sigma_{s}\left(\rho \sigma_{c}-\sigma_{s}\right)\right\}
$$

For this ratio to equal unity, the term in brackets on the right hand side must be zero, so that:

$$
\rho \frac{\sigma_{c}}{\sigma_{s}}=1
$$

And since $\rho=\frac{\sigma_{c s}}{\sigma_{c} \sigma_{s}}$ and $\gamma=\frac{\sigma_{\mathrm{cs}}}{\sigma_{\mathrm{s}}^{2}}$ where $\gamma$ is the regression coefficient in a linear regression of the $\log$ of $c$ on the $\log$ of $s$, the condition applies only if the regression coefficient is unity. This condition is in fact equivalent, except for the addition of a random error term, to the assumption that the ratio $c / s$ is the same for all age groups, since there is a unit elasticity of $c$ with respect to $s$. This assumption is clearly very strong.

However, there is an alternative case, under which the two terms (the ratio of averages and the average of ratios) are not equal, but if the two variables are independent, so that $\rho=0$, the ratio of averages is a proportion of the average of ratios, with the constant of proportionality being equal to $\exp \left(-\sigma_{s}^{2}\right)$. It is not unreasonable to suppose that this term is constant over time. 


\section{REFERENCES}

Atkinson, A.B. (1970) On the Measurement of Inequality. Journal of Economic Theory, 2, pp. 244-63.

Australian Government (2002) Intergenerational Report. 2002-2003 Budget Paper No. 5. Commonwealth of Australia, Canberra.

Banks, J. and Johnson, P. (1994) Equivalence scale relativities revisited. Economic Journal, 104, pp. 883-890.

Barro, R. (1979) On the Determination of Public Debt. Journal of Political Economy 87, pp. $940-71$

Barro, R. and Sala-I-Martin, X. (1995) Economic Growth. New York: McGraw-Hill.

Carroll, C. D., Overland, J. and Weil, D, N. (1997) Comparison utility in a growth model. Journal of Economic Growth, 2, pp. 339-367

Chamley, C. (1986) Optimal taxation of capital income in general equilibrium with infinite lives. Econometrica, 54, pp. 607-622

Creedy, J. (2006) Evaluating Policy: Welfare Weights and Value Judgements. University of Melbourne Department of Economics Working Paper,

Creedy, J. and Sleeman, C. (2005) Adult equivalence scales, inequality and poverty. New Zealand Economic Papers, 39, pp. 51-83.

Cutler, D. M. and Katz, L. (1992) Rising inequality? Changes in the distribution of income and consumption in the 1980s. American Economic Review, 82, pp. 546-551.

Decoster, A. and Ooghe, E. (2003) Weighting with individuals, equivalent individuals or not weighting at all: does it matter empirically?. In Inequality, Welfare and Poverty: Theory and Measurement (ed. by Y. Amiel and J.A. Bishop), pp. 173-190. New York: Elsevier.

Ebert, U. (1997) Social welfare when needs differ: an axiomatic approach. Economica, 64, pp. 233-244.

Faruqee, H. and Muhleisen, M. (2001) Population Ageing in Japan: Demographic Shock and Fiscal Sustainability. International Monetary Fund Working Paper, no. $01 / 40$. 
Glewwe, P. (1991) Household equivalence scales and the measurement of inequality: transfers from the poor to the rich could decrease inequality. Journal of Public Economics, 44, pp. 214-216.

Jenkins, S.P. and Cowell, F.A. (1994) Parametric equivalence scales and scale relativities. Economic Journal, 104, pp. 891-900.

Kirman, P. (1992) Whom or what does the representative individual represent? Journal of Economic Perspectives, 6, pp. 117-136

Lewbel, A. (1989) Exact aggregation and a representative consumer. Quarterly Journal of Economics, 104, pp. 622-633.

Obstfeld, M. and Rogoff, K. (1996) Foundations of International Macroeconomics. London: The MIT Press.

Productivity Commission (2005) Economic Implications of an Ageing Australia. Canberra: Commonwealth of Australia.

Ramsey, F. (1928) A mathematical theory of saving. Economic Journal, 38, pp.543559.

Shorrocks, A.F. (2004) Inequality and welfare evaluation of heterogeneous income distributions. Journal of Economic Inequality, 2, pp. 193-218.

Turner, D., Giorno, C. DeSerres, A., Vourch, A. and Richardson, P. (1998) The Macroeconomic Implications of Ageing in a Global Context. OECD Economic Department Working Paper, no. 193.

Werner, R. and Veld, J. (2002) Some Selected Simulation Experiments with the European Commission's QUEST model. European Commission, Brussels. 